\title{
Coexistence of chiral symmetry restoration and random orientation of galaxies
}

\author{
B. Aryal ${ }^{1,2}$, S. Paudel ${ }^{2}$, and W. Saurer ${ }^{1}$ \\ 1 Institut für Astrophysik, Universität Innsbruck, Technikerstraße 25, 6020 Innsbruck, Austria \\ e-mail: [binil.aryal; walter.saurer]@uibk.ac.at \\ 2 Central Department of Physics, Tribhuvan University, Kirtipur, Kathmandu, Nepal \\ e-mail: sanjpaudel@yahoo.com
}

Received 8 May 2007 / Accepted 21 November 2007

\section{ABSTRACT}

\begin{abstract}
We studied the chiral symmetry restoration and the spatial orientation of 2288 spiral and spiral barred galaxies that have radial velocities (RV) less than $5000 \mathrm{~km} \mathrm{~s}^{-1}$. A random direction of the rotation of galaxies is assumed in order to classify the structural modes. The distribution of spin vector and spin vector projections of leading and trailing arm galaxies in the total sample and subsamples are studied. We use chi-square, auto-correlation and Fourier tests in order to discriminate the preferred alignments from the random alignments. A good correlation between the random alignment and the chiral symmetry is noticed in the Local Supercluster $\left(\mathrm{RV}<3000 \mathrm{~km} \mathrm{~s}^{-1}\right)$ and in galaxies nearby the Local Supercluster $\left(3000<\mathrm{RV}\left(\mathrm{km} \mathrm{s}^{-1}\right) \leq 5000\right)$. Spiral galaxies show a similar result. The barred spirals show an opposite trend to that observed for the spirals. Nearby the Local Supercluster, we noticed a preferred spatial alignment and non-chiral property in the leading and trailing arm spiral barred galaxies. Our result predicts that the progressive loss of chirality might have some connection with the rotationally supported (spirals, barred spirals) and randomized (lenticulars, ellipticals) systems. Thus, we suspect that the dynamical processes in the cluster evolution give rise to a dynamical loss of chirality.
\end{abstract}

Key words. Galaxy: formation - galaxies: statistics - Galaxy: evolution - Galaxy: structure

\section{Introduction}

An object is said to be chiral if it cannot be superposed on its mirror image, such as the letter " $\mathrm{B}$ " and its mirror image. These objects do not show reflection symmetry, but may exhibit rotational symmetry. In quantum field theory, chiral symmetry is a possible symmetry of the Lagrangian, under which the lefthanded and right-handed parts of Dirac fields transform independently. The chiral symmetry transformation can be divided into a component that treats the left-handed and the right-handed parts equally, known as vector symmetry, and a component that treats them differently, known as axial symmetry. A non-chiral object is called achiral and can be superimposed on its mirror image. Chiral property is a key ingredient in different problems of theoretical physics, from nonperturbative quantum cromodynamics to highly doped semiconductors (Garcia-Garcia \& Cuevas 2006). For a pseudoscalar meson, it is found that the chiral symmetry breaking decreases with increasing current-quark mass (Chang et al. 2007). Bagchi et al. (2006) studied the large color approximation of the compact strange stars and discussed the chiral symmetry restoration. They claimed that the chiral symmetry restoration can be understood by exploring the possible existence of strange stars in the Universe.

In principle, initial quantum fluctuations have been hugely enhanced during the inflationary epoch, leading to the formation of the large scale structure in the Universe (Peacock 1999; Liddle \& Lyth 2000). Thus, the macroscopic chirality should be related to some primordial microscopic process, which has led to the large scale structures observed today (Fall 1992). It is therefore interesting to study the existence of chiral symmetry not only in microscopic phenomena (L-neutrino, mesons, L-aminoacids, D-sugars, etc.), but also in macroscopic ones (stars, clouds, galaxies, etc.).

By considering the group of transformations acting on the configuration space, Capozziello \& Lattanzi (2006) claimed that the spiral galaxies exhibit chiral symmetry in the large scale structure. In addition, they predicted that the progressive loss of chirality might have some connection with the rotationally-supported (spirals, barred spirals) and randomized systems (lenticulars, ellipticals). Aryal et al. (2007a) carried out a study to test Capozziello \& Lattanzi's (2006) prediction regarding the progressive loss of chirality in the large scale structure. They concluded the existence of chiral symmetry for both the spirals and the barred spirals in the Local Supercluster (LSC). However, the Virgo cluster galaxies show a preferred alignment: the galactic rotation axes of leading and trailing structures are found to lie in the equatorial plane. Aryal \& Saurer (2005a) noticed a preferred alignment for the late-type spirals and barred spirals in the LSC. In addition, they found that the spin vector (SV) projections of early- and late-type spirals show opposite alignment. Their results hint the existence of the chiral characterization.

In this work, we study the chiral symmetry and the spatial orientation of the galaxies having radial velocity $(\mathrm{RV}) \leq 5000 \mathrm{~km} \mathrm{~s}^{-1}$. We intend to study the correlation between the chiral symmetry breaking and the preferred alignment of galaxies in the total sample and subsamples. In addition, we ask the following questions: (1) does chirality exist for the spirals and barred spirals in our database; (2) does morphological dependence exist concerning the spatial orientation of leading and 
trailing arm galaxies; (3) do the subsamples of LSC and nearby LSC galaxies exhibit chiral symmetry, (4) is there any correlation between the chirality and the preferred alignment of galaxies; and (5) what can we say about the chiral and achiral (nonchiral) properties of the large scale structure.

First, in Sect. 2 we review past literatures concerning the structural modes of the spiral galaxies. The data reduction procedure and method of analysis are described in Sects. 3 and 4. A discussion of the results concerning chiral symmetry and the preferred spatial alignment is presented in Sects. 5 and 6. Finally, we present the discussion, compare the results with the previous works and draw conclusions in Sects. 7 and 8. Three appendices concerning the determination of the SV and SV projections of galaxies (A), removal of selection effects (B) and the statistics $(\mathrm{C})$ are added.

\section{Winding sense of spirals}

In order to understand true structural modes (leading or trailing) of spiral galaxies, we need to know the direction of the spiral pattern (S- or Z-shaped), the approaching and receding sides and the near and far parts, since galaxies are commonly inclined in space to the line of the sight. The $\mathrm{S}$ and Z-patterns can be determined from the image of the galaxy. Similarly, the approaching and receding sides can be defined if spectroscopic data on rotation is available. Determining the near and far parts, however, is fairly hard to establish. For this, Pasha (1985) used "tilt" criteria and studied the sense of winding of the arms in 132 spirals. He found 107 spirals to have trailing arms. It should be remembered that the classical "tilt" criteria is based on the visible asymmetry of a dust matter distribution. It is well known that the dark matter (DM) halo generally dominates the dynamics of the galaxies. Ostriker et al. (1974) predicted that the DM is concentrated in the extended galactic halos. A significant role of DM halos is expected by the standard CDM model of hierarchical galaxy formation. In our own galaxy, the observed rotation of the stars and gas clouds indicates that the visible matter is surrounded by a halo of this DM containing the major portion of the total galaxy mass and extending very far beyond the visible matter (Olling $\&$ Merrifield 2000). The nature of DM in the galactic halo of spiral galaxies is still undetermined. Thomasson et al. (1989) studied theoretically and performed $N$-body simulations in order to understand the formation of spiral structures in retrograde galaxy encounters. Interestingly, they noticed the importance of halo mass. They concluded that the spirals that have halos with masses larger than the disk mass exhibit leading pattern.

Differential rotation in a galaxy's disc generates density waves in the disc, leading to spiral arms. According to gravitational theory, the spiral arms are born as leading and subsequently transform to trailing modes. With the passage of time, the spiral pattern deteriorates gradually by the differential rotation of the plane of the galaxy, but the bar structure persists for a long time (Oort 1970a). This structure can again regenerate spiral patterns in the outer region. Thus, a close relation between the origin of the leading and the trailing arm of the galaxies cannot be denied (Oort 1970b). Thus, the makeup of galactic halos is important to cosmology in order to understand the winding sense of spiral galaxies.

Sugai \& Iye (1995) used statistics to study the winding sense of galaxies (S- and Z-shaped) in 9825 spirals. No significant dominance from a random distribution is noticed. Aryal \& Saurer (2005a) studied the spatial orientations of SVs of 4073 galaxies in the LSC. No preferred alignment is found. These results hint that the distribution of angular momentum of galaxies is entirely random in two- ( $\mathrm{S}$ - and Z-shaped) and threedimensional (SV) analysis, provided the database is rich. Thus, one can assume that the choice of rotation for a galaxy might be random for an observer.

\section{The sample: data reduction}

We used the NASA Extragalactic Database (http://nedwww. ipac.caltech.edu/) to compile the sample galaxies. The selection criteria were: (1) RV of the galaxy $<5000 \mathrm{~km} \mathrm{~s}^{-1}$, (2) the morphology should be defined, (3) the diameters, magnitude and the position angle should be given, and (4) the arm patterns (Sor Z-shaped) should be visible.

The galaxies of the clusters A0426 (mean RV $5366 \mathrm{~km} \mathrm{~s}^{-1}$, $75 \pm 5 \mathrm{Mpc}$ ) and A3627 (mean RV $4881 \mathrm{~km} \mathrm{~s}^{-1}, 63 \pm 4 \mathrm{Mpc}$ ) are superimposed in our database (Abell et al. 1989; Struble $\&$ Rodd 1999). We removed these cluster galaxies using Brunzendorf \& Meusinger (1999), Photometric Atlas of Northern Bright Galaxies (Kodaira et al. 1990) and the Uppsala Galaxy Catalogue (Nilson 1973).

Las Campanas Redshift Survey (Shectman 1996) is used to compile the RVs of the galaxies. The position angle (PA) and the diameters of galaxies were added from the Uppsala Galaxy Catalogue (Nilson 1973), Uppsala obs. General Catalogue, Addendum (Nilson 1974), Photometric Atlas of Northern Bright Galaxies (Kodaira et al. 1990), ESO/Uppsala Survey of the European Southern Observatory (Lauberts 1982), Southern Galaxy Catalogue (Corwin et al. 1985) and Third Reference Catalogue of Bright Galaxies (de Vaucouleurs et al. 1991).

The determination of the direction of rotation of the galaxies is a difficult problem. In order to determine true rotation, huge spectroscopic data is needed. In addition, one should identify which side of the galaxy is towards and which side is far from us. These data were available only for a few galaxies. The direction of rotation and the arm patterns ( $\mathrm{S}$ - and $\mathrm{Z}$-shaped) should be known in order to distinguish the structural modes (leading and trailing) of a spiral galaxy. The leading mode is one whose outer tip points towards the direction of galactic rotation (see Fig. 1a). Similarly, the outer tip of trailing mode directs in the direction opposite to the galactic rotation. The arm patterns (Sor Z-shaped) can be studied from the images of the galaxies. Without knowing actual rotation, it is impossible to identify the true mode. We assume that the choice of rotation of an individual galaxy is random for a observer. We used computer to give random directions to our galaxies. Thus, each galaxy got a virtual direction by the computer. In this way, we identified structural modes of galaxies in our database. For individual galaxies, these structural modes might be incorrect. As a whole, it certainly reveals the secret (to some extent) in the subjective sense. In addition, we have information regarding the morphology, diameters, inclination angle, RVs, and magnitudes of individual galaxies in order to study true spatial orientations of SVs of galaxies.

In this way, we compiled a database of 2288 galaxies, which we classified into two groups: leading and trailing. An example of "S" and "Z" patterns are shown in Fig. 1b. All sky distribution of leading and trailing patterns is shown in Fig. 2a. We further classify the total galaxies into LSC $\left(\mathrm{RV} \leq 3000 \mathrm{~km} \mathrm{~s}^{-1}\right)$ and nearby LSC $\left(3000<\mathrm{RV}\left(\mathrm{km} \mathrm{s}^{-1}\right) \leq 5000\right)$ galaxies. Our database includes 667LSC and 1621 nearby LSC galaxies. These galaxies are shown in Figs. 2b, c.

Several groups and aggregations of the galaxies can be seen in the all-sky distribution of LSC and nearby LSC galaxies (Figs. 2b, c) The inhomogeneous distribution of the positions 

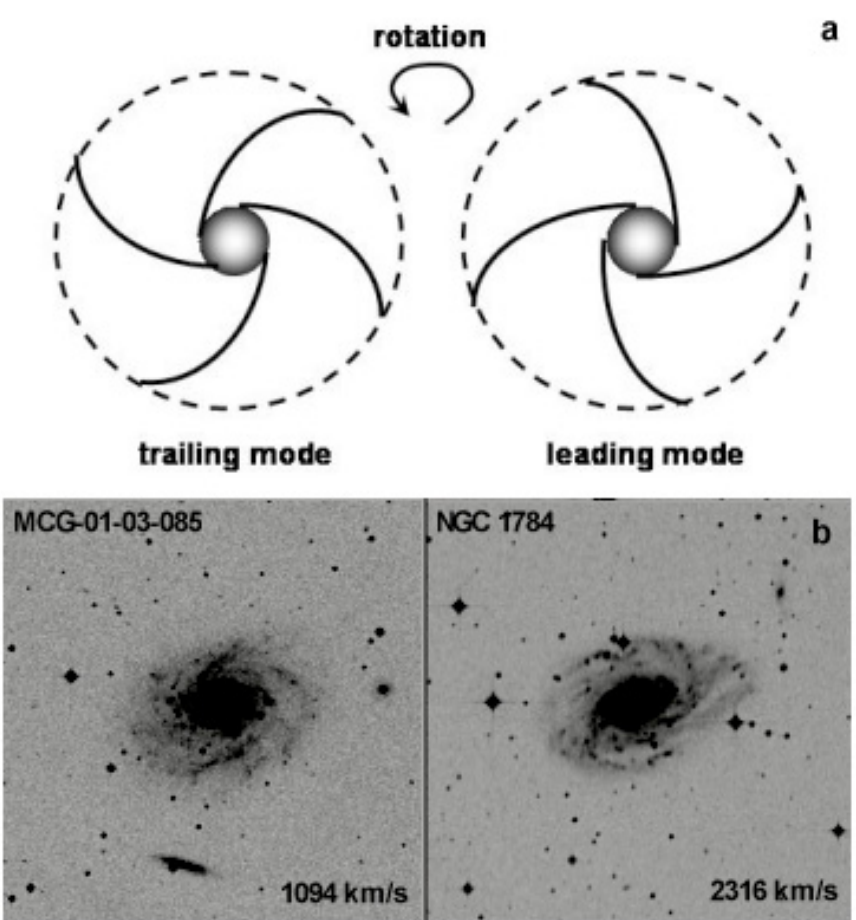

Fig. 1. a) Trailing and leading structures of spiral galaxies. Spiral arms can be distinguished according to their orientation relative to the direction of rotation of the galaxy. The outer tip of a leading arm galaxy points towards the direction of galactic rotation, whereas the trailing arm is one whose outer tip directs in the direction opposite to the galactic rotation. b) An example of "S" and " $Z$ " arm pattern galaxies in our database. The name and the RV of the galaxies are given in the image.

of the galaxies might be the selection effects for the galaxy orientation study (Aryal \& Saurer 2000, 2001).

The inclination angle $(i)$ of galaxies was estimated using Holmberg's (1946) formula: $\cos ^{2} i=\left[(b / a)^{2}-q^{2}\right] /\left(1-q^{2}\right)$, with $b / a$ the measured axial ratio and $q$ the intrinsic flatness. We used the intrinsic flatness value as suggested by Haynes \& Giovanelli (1984) and estimated the inclination angle of our galaxies. Haynes \& Giovanelli (1984) suggested different $q$ values for different types of galaxies, ranging from $q=0.23$ (for ellipticals) to $q=0.10$ (for late-type spirals). We used $q=0.20$ for morphologically unidentified galaxies.

The RV, inclination angle, major diameter and the magnitude distributions of the leading and trailing arm LSC and nearby LSC galaxies are shown in Fig. 3. In the histogram, the greyshaded bar and the solid circle with error bars represent the number of leading and trailing arm galaxies, respectively.

The database of LSC galaxies is dominated by the galaxies that have $\mathrm{RV} \geq 1200 \mathrm{~km} \mathrm{~s}^{-1}$ whereas the database of nearby LSC galaxies is found to be homogeneous (Figs. 3a, b). The inclination angle distribution shows a good agreement with the expected cosine distribution in the limit $75^{\circ} \leq i \leq 35^{\circ}$ (Figs. 3c, d). The lack of low inclination angle galaxies can be seen in Figs. 3c, d. This is the major selection effect in our database. In our database, the preferred size (i.e., major diameter) of the LSC and nearby LSC galaxies are found 1-4 arcmin and 0.5-2 arcmin, respectively (Figs. 3e, f). Similarly, the majority of LSC and nearby LSC galaxies have magnitudes in the range 11-15 and 13-16, respectively (Figs. 3g, h).

We classified the database of LSC and nearby LSC galaxies into 25 and 30 subsamples for both the leading and trailing
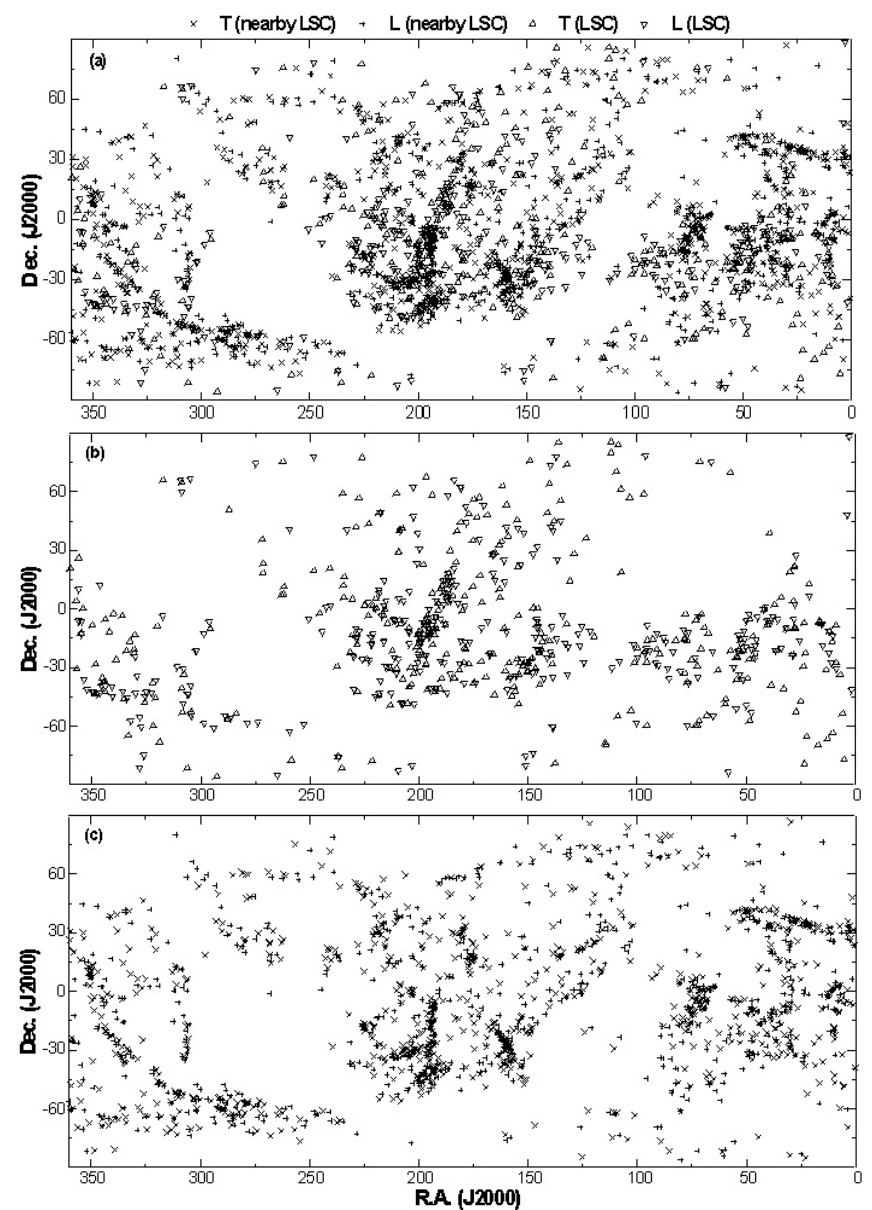

Fig. 2. All-sky distribution of the total a), LSC $\left(\mathrm{RV}<3000 \mathrm{~km} \mathrm{~s}^{-1}\right)$ b), and nearby LSC galaxies $\left(3000 \leq \mathrm{RV}\left(\mathrm{km} \mathrm{s}^{-1}\right)<5000\right)$ c). The symbols " $\times$ " ("+") and " $\triangle$ " (" $\nabla ")$ represent the trailing (leading) patterns in the nearby LSC and LSC galaxies, respectively.

modes on the basis of the morphology, RV, inclination angle, major diameter and magnitude of galaxies. The chiral properties of the galaxies in these subsamples are studied in Sect. 4.

\section{Method of analysis}

\subsection{Chiral symmetry}

For a subsample to show chiral symmetry, we set the difference between the number of leading and trailing modes, $\Delta \leq 5 \%$. The chiral symmetry is assumed to be violated when $\Delta>5 \%$.

We classify the total sample into various subsamples on the basis of the morphology, RV, inclination angle, major diameters and the magnitude of the galaxies. Basic statistics is used to study the difference between the number of leading and trailing arm galaxies in the subsample.

Thus, the preferred structural modes (leading or trailing arm) are studied in the subsamples of LSC $\left(\mathrm{RV}<3000 \mathrm{~km} \mathrm{~s}^{-1}\right)$, nearby LSC $\left(3000<\mathrm{RV}\left(\mathrm{km} \mathrm{s}^{-1}\right) \leq 5000\right)$ and total $(\mathrm{RV}<$ $5000 \mathrm{~km} \mathrm{~s}^{-1}$ ) galaxies, separately. Finally, possible explanations of the dominance of leading or trailing arm galaxies in the subsamples are discussed. In addition, the chirality loss sequence will be explained. 

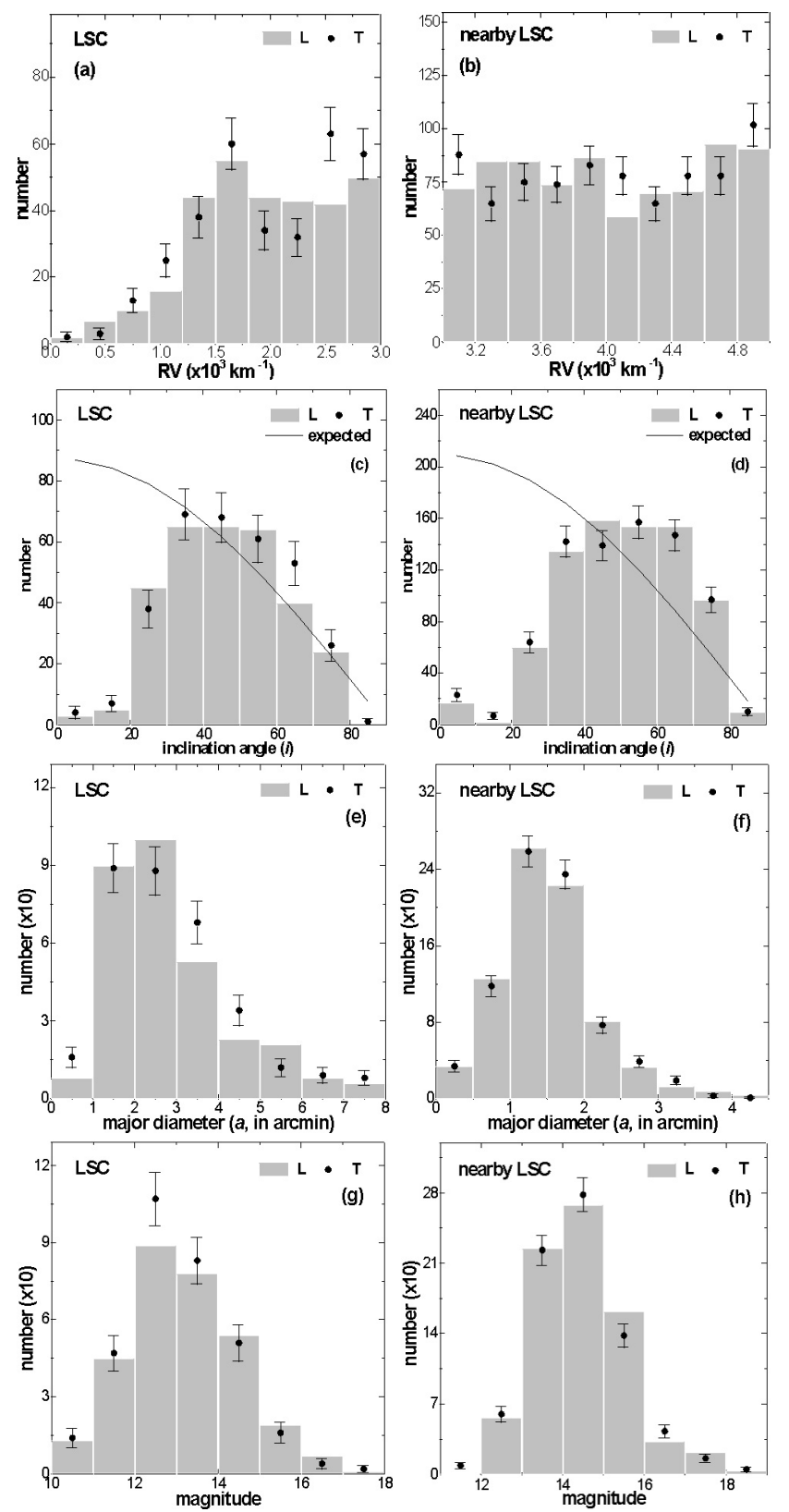

Fig. 3. The RV a), b), inclination angle c), d), major diameter e), f) and the magnitude distribution $\mathbf{g}$ ), $\mathbf{h}$ ) of the leading and the trailing patterns in the LSC and nearby LSC galaxies. The statistical $\pm 1 \sigma$ error bars are shown for the trailing $(\bullet)$ subsample. The solid line c), d) represents the expected distribution.

\subsection{Preferred alignment}

We assume an isotropic distribution as a theoretical reference to study the preferred orientation in the LSC $\left(\mathrm{RV}<3000 \mathrm{~km} \mathrm{~s}^{-1}\right)$, nearby LSC $\left(3000<\mathrm{RV}\left(\mathrm{km} \mathrm{s}^{-1}\right) \leq 5000\right)$ and total $\left(\mathrm{RV}<5000 \mathrm{~km} \mathrm{~s}^{-1}\right)$ galaxies, separately. For this, we use "PA-inclination" method as proposed by Flin \& Godlowski (1986) in order to convert two dimensional parameters (i.e., PA) into three dimensional parameters (i.e., polar and azimuthal angles) (Appendix A). The selection effects in the database are removed and the expected isotropic distribution for polar $(\theta)$ and azimuthal $(\phi)$ angles are determined using the method proposed by Aryal \& Saurer (2000) (Appendix B). We use the supergalactic coordinate system as a reference. In order to measure the deviation from the isotropic distribution we carried out
Table 1. Statistics of leading (Col. 3) and trailing (Col. 4) patterns in the LSC, nearby LSC and total (LSC + nearby LSC) galaxies. The fifth column gives the percentage difference $(\Delta=(T-L) /(T+L) \times 100)$ between the trailing $(T)$ and the leading $(L)$ structural modes. The last column gives the error: $\sigma(\%)=(\sqrt{T}-\sqrt{L}) /(\sqrt{L}+\sqrt{T}) \times 100$. The sample/subsample and their abbreviations are given in the first two columns.

\begin{tabular}{lcccrr}
\hline \hline Subsample & Symbol & $L$ & $T$ & $\Delta(\%)$ & $\sigma(\%)$ \\
\hline LSC galaxies & & & & & \\
total & $\mathrm{T} 1$ & 319 & 348 & -4.3 & -2.2 \\
spirals & $\mathrm{S} 1$ & 153 & 179 & -7.8 & -3.9 \\
spiral barred & $\mathrm{SB} 1$ & 146 & 151 & -1.7 & -0.8 \\
others & $\mathrm{O} 1$ & 20 & 18 & 5.3 & 2.6 \\
\hline nearby LSC galaxies & & & & & \\
total & $\mathrm{T} 2$ & 814 & 807 & 0.4 & 0.2 \\
spirals & $\mathrm{S} 2$ & 395 & 367 & 3.7 & 1.8 \\
spiral barred & $\mathrm{SB} 2$ & 191 & 269 & -17.0 & -8.5 \\
others & $\mathrm{O} 2$ & 31 & 37 & -8.8 & -4.4 \\
\hline LSC + nearby LSC & & & & & \\
total & $\mathrm{T} 3$ & 1133 & 1155 & -1.0 & -0.5 \\
spirals & $\mathrm{S} 3$ & 548 & 546 & 0.2 & 0.1 \\
spiral barred & $\mathrm{SB} 3$ & 337 & 420 & -11.0 & -5.5 \\
others & $\mathrm{O} 3$ & 51 & 55 & -3.8 & -1.9 \\
\hline
\end{tabular}

three statistical tests: chi-square, auto-correlation and the Fourier (Appendix C).

The polar and azimuthal angle distributions in the 10 sample and subsamples of the leading and trailing arm LSC, nearby LSC and total galaxies are studied.

\section{Chiral symmetry}

Table 1 summarizes of the database of leading and trailing arm galaxies in the LSC and nearby LSC galaxies compiled by Aryal et al. (2007a, present work). The last four rows give a brief statistics of the leading and trailing arm galaxies that have $\mathrm{RV} \leq 5000 \mathrm{~km} \mathrm{~s}^{-1}$ (LSC + nearby LSC, total hereafter).

The percentage differences $(\Delta \%)$ between the total leading and trailing arm galaxies are found to be less than 5\% in the LSC (subsample T1), nearby LSC (subsample T2) and total galaxies (subsample T3). Aryal et al. (2007a) noticed chiral symmetry violation in the Virgo cluster region. This violation leads the LSC spirals (S1) to give $\Delta>5 \%$. In addition, $\Delta$ value is found to be $>5 \%$ in the subsamples $\mathrm{O} 1, \mathrm{SB} 2, \mathrm{O} 2$ and SB3. It seems that the SB galaxies do not exhibit chiral symmetry.

We study the inclination angle, RV, magnitude and major diameter dependence concerning the chiral symmetry in the LSC, nearby LSC and total galaxies (Table 2). At the end, a general discussion will be presented.

\section{1. $L S C$ galaxies $\left(R V \leq 3000 \mathrm{~km} \mathrm{~s}^{-1}\right)$}

Figure 4 shows the comparison between the leading and trailing arm galaxies in the subsamples as listed in Table 2 . The $\Delta(\%)$ in Table 2 and Fig. 4 represent the percentage difference between the number of trailing and leading arm galaxies. In Fig. 4, the grey-shaded region corresponds to the region showing $\Delta \%$ value $\leq 5 \%$.

Out of 22,12 subsamples (54\%) show the $\Delta \%$ value $> \pm 5 \%$. Seven subsamples give the $\Delta \%$ value $> \pm 10 \%$. A significant dominance $(\Delta \%=-20 \%)$ of trailing structural mode is noticed for the galaxies that have RVs 2400 to $2700 \mathrm{~km} \mathrm{~s}^{-1}$ (Table 2). 
Table 2. Statistics of leading and trailing patterns in the subsamples of LSC, nearby LSC and total (LSC + nearby LSC) galaxies. The sample/subsample and their abbreviations are given in the first two columns. The symbols RV, $i, a$ and $m$ represent the radial velocity, inclination angle, major diameter and the magnitude of galaxies. Other symbols and the explanations are as in Table 1.

\begin{tabular}{|c|c|c|c|c|c|}
\hline Subsample & Symbol & $L$ & $T$ & $\Delta(\%)$ & $\sigma(\%)$ \\
\hline \multicolumn{6}{|l|}{ LSC galaxies } \\
\hline $20^{\circ} \leq i<30^{\circ}$ & I1(1) & 45 & 38 & 8.4 & 4.2 \\
\hline $30^{\circ} \leq i<40^{\circ}$ & $\mathrm{I} 2(2)$ & 65 & 69 & -3.0 & -1.5 \\
\hline $40^{\circ} \leq i<50^{\circ}$ & I3(3) & 65 & 68 & -2.3 & -1.1 \\
\hline $50^{\circ} \leq i<60^{\circ}$ & I4(4) & 64 & 61 & 2.4 & 1.2 \\
\hline $60^{\circ} \leq i<70^{\circ}$ & I5(5) & 40 & 53 & -14.0 & -7.0 \\
\hline $70^{\circ} \leq i<80^{\circ}$ & I6(6) & 24 & 26 & -4.0 & -2.0 \\
\hline $\mathrm{RV}<1200 \mathrm{~km} \mathrm{~s}^{-1}$ & RV1(7) & 35 & 43 & -10.3 & -5.1 \\
\hline $1200 \leq \mathrm{RV}<1500$ & RV2(8) & 44 & 38 & 7.3 & 3.7 \\
\hline $1500 \leq \mathrm{RV}<1800$ & RV3(9) & 55 & 60 & -4.3 & -2.2 \\
\hline $1800 \leq \mathrm{RV}<2100$ & RV4(10) & 44 & 34 & 12.8 & 6.4 \\
\hline $2100 \leq \mathrm{RV}<2400$ & RV5(11) & 43 & 32 & 14.7 & 7.4 \\
\hline $2400 \leq \mathrm{RV}<2700$ & RV6(12) & 42 & 63 & -20.0 & -10.1 \\
\hline $2700 \leq \mathrm{RV}<3000$ & RV7(13) & 50 & 57 & -6.5 & -3.3 \\
\hline$m<12$ & M1(14) & 45 & 47 & -2.2 & -1.1 \\
\hline $12 \leq m<13$ & M2(15) & 89 & 107 & -9.2 & -4.6 \\
\hline $13 \leq m<14$ & M3(16) & 78 & 83 & -3.1 & -1.6 \\
\hline $14 \leq m<15$ & M4(17) & 54 & 51 & 2.9 & 1.4 \\
\hline$m>15$ & M5(18) & 27 & 22 & 10.2 & 5.1 \\
\hline$a<2^{\prime}$ & A1(19) & 98 & 105 & -3.4 & -1.7 \\
\hline $2^{\prime} \leq a<3^{\prime}$ & $\mathrm{A} 2(20)$ & 100 & 88 & 6.4 & 3.2 \\
\hline $3^{\prime} \leq a<4^{\prime}$ & $\mathrm{A} 3(21)$ & 53 & 68 & -12.4 & -6.2 \\
\hline$a \geq 4^{\prime}$ & $\mathrm{A} 4(22)$ & 58 & 63 & -4.1 & -2.1 \\
\hline \multicolumn{6}{|l|}{ nearby LSC galaxies } \\
\hline $20^{\circ} \leq i<30^{\circ}$ & $\mathrm{I} 7(23)$ & 60 & 64 & -3.2 & -1.6 \\
\hline $30^{\circ} \leq i<40^{\circ}$ & I8(24) & 135 & 142 & -2.5 & -1.3 \\
\hline $40^{\circ} \leq i<50^{\circ}$ & $\mathrm{I} 9(25)$ & 159 & 139 & 6.7 & 3.4 \\
\hline $50^{\circ} \leq i<60^{\circ}$ & I10(26) & 154 & 157 & -1.0 & -0.5 \\
\hline $60^{\circ} \leq i<70^{\circ}$ & I11(27) & 154 & 147 & 2.3 & 1.2 \\
\hline $70^{\circ} \leq i<80^{\circ}$ & $\mathrm{I} 12(28)$ & 97 & 97 & 0.0 & 0.0 \\
\hline $3000 \leq \mathrm{RV}<3200$ & RV8(29) & 72 & 88 & -10.0 & -5.0 \\
\hline $3200 \leq \mathrm{RV}<3400$ & RV9(30) & 85 & 65 & 13.3 & 6.7 \\
\hline $3400 \leq \mathrm{RV}<3600$ & RV10(31) & 85 & 75 & 6.3 & 3.1 \\
\hline $3600 \leq \mathrm{RV}<3800$ & RV11(32) & 74 & 74 & 0.0 & 0.0 \\
\hline $3800 \leq \mathrm{RV}<4000$ & RV12(33) & 87 & 83 & 2.4 & 1.2 \\
\hline $4000 \leq \mathrm{RV}<4200$ & RV13(34) & 59 & 78 & -13.9 & -7.0 \\
\hline $4200 \leq \mathrm{RV}<4400$ & RV14(35) & 70 & 65 & 3.7 & 1.9 \\
\hline $4400 \leq \mathrm{RV}<4600$ & RV15(36) & 71 & 78 & -4.7 & -2.4 \\
\hline $4600 \leq \mathrm{RV}<4800$ & RV16(37) & 93 & 78 & 8.8 & 4.4 \\
\hline $4800 \leq \mathrm{RV}<5000$ & RV17(38) & 91 & 102 & -5.7 & -2.9 \\
\hline$m<13$ & M6(39) & 57 & 60 & -2.6 & -1.3 \\
\hline $13 \leq m<14$ & M7(40) & 225 & 223 & 0.4 & 0.2 \\
\hline $14 \leq m<15$ & M8(41) & 268 & 278 & -1.8 & -0.9 \\
\hline $15 \leq m<16$ & M9(42) & 162 & 138 & 8.0 & 4.0 \\
\hline$m>16$ & M10(43) & 55 & 59 & -3.5 & -1.8 \\
\hline$a<1^{\prime}$ & A5(44) & 34 & 34 & 0.0 & 0.0 \\
\hline $1^{\prime} \leq a<1.5^{\prime}$ & A6(45) & 126 & 118 & 3.3 & 1.6 \\
\hline $1.5^{\prime} \leq a<2^{\prime}$ & A7(46) & 263 & 259 & 0.8 & 0.4 \\
\hline $2^{\prime} \leq a<2.5^{\prime}$ & A8(47) & 224 & 235 & -2.4 & -1.2 \\
\hline $2.5^{\prime} \leq a<3^{\prime}$ & A9(48) & 81 & 77 & 2.5 & 1.3 \\
\hline$a>3^{\prime}$ & A10(49) & 58 & 62 & -3.3 & -1.7 \\
\hline \multicolumn{6}{|l|}{ LSC + nearby LSC } \\
\hline $20^{\circ} \leq i<30^{\circ}$ & $\mathrm{I} 13(50)$ & 105 & 102 & 1.4 & 0.7 \\
\hline $30^{\circ} \leq i<40^{\circ}$ & $\mathrm{I} 14(51)$ & 200 & 211 & -2.7 & -1.3 \\
\hline $40^{\circ} \leq i<50^{\circ}$ & $\mathrm{I} 15(52)$ & 224 & 207 & 3.9 & 2.0 \\
\hline $50^{\circ} \leq i<60^{\circ}$ & I16(53) & 218 & 218 & 0.0 & 0.0 \\
\hline $60^{\circ} \leq i<70^{\circ}$ & $\mathrm{I} 17(54)$ & 194 & 200 & -1.5 & -0.8 \\
\hline $70^{\circ} \leq i<80^{\circ}$ & $\mathrm{I} 18(55)$ & 121 & 123 & -0.8 & -0.4 \\
\hline$m<13$ & M11(56) & 191 & 214 & -5.7 & -2.8 \\
\hline $13 \leq m<14$ & M12(57) & 303 & 306 & -0.5 & -0.2 \\
\hline $14 \leq m<15$ & M13(58) & 322 & 329 & -1.1 & -0.5 \\
\hline$m>15$ & M14(59) & 244 & 219 & 5.4 & 2.7 \\
\hline$a<2^{\prime}$ & A10(60) & 521 & 516 & 0.5 & 0.2 \\
\hline $2^{\prime} \leq a<3^{\prime}$ & A11(61) & 405 & 400 & 0.6 & 0.3 \\
\hline$a>3^{\prime}$ & A12(62) & 169 & 193 & -6.6 & -3.3 \\
\hline
\end{tabular}

Leading modes dominate $(\Delta>12 \%)$ for the galaxies that have RVs 1800 to $2400 \mathrm{~km} \mathrm{~s}^{-1}$. Interestingly, these subsamples (RV4,
RV5, RV6) compensate each other to give $\Delta$ value $0 \%$, suggesting the existence of chiral symmetry in high RV LSC galaxies. 


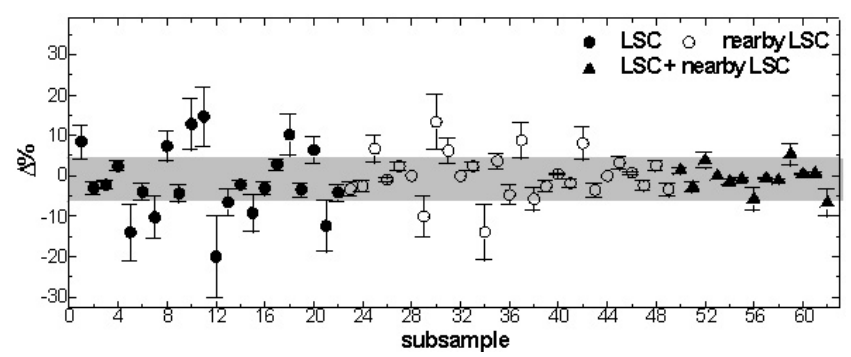

Fig. 4. The basic statistics of the leading and trailing patterns in the subsamples. The $X$-axis represents the number corresponding to the subsample listed in the second column of Table 2. See Table 1 for the explanation of $\Delta$. The grey-shaded region $(\Delta \leq \pm 5 \%)$ represents the region of chiral symmetry.

However, the chiral symmetry violation cannot be denied in the subsamples.

The dominance $(\Delta \%> \pm 8 \%)$ of leading and trailing structural modes is noticed for low $\left(20^{\circ} \leq i<30^{\circ}\right)$ and high inclination $\left(60^{\circ} \leq i<70^{\circ}\right)$ galaxies, respectively. Similar to this result, the low $(m<14)$ and high magnitude $(m>14)$ galaxies show an excess number of trailing and leading arm galaxies, respectively. A dominance of trailing structural modes is found for the galaxies that have $a>3$ arcmin. These findings are interesting in the context of the Virgo cluster.

Aryal et al. (2007a) noticed two groups of galaxies in the Virgo cluster region. In these groups, the dominance of trailing and leading structural modes are found. They concluded that the aggregation of the leading and trailing arm galaxies might have already started in the Virgo cluster region. This process might lead to the violation of chiral symmetry in the subsamples.

\subsection{Nearby LSC galaxies $\left(3000<R V\left(\mathrm{~km} \mathrm{~s}^{-1}\right) \leq 5000\right)$}

Seven subsamples $(26 \%)$ of nearby LSC galaxies have an $\Delta \%$ value $> \pm 5 \%$. These galaxies are not the cluster galaxies. Thus, the chiral symmetry is found to be stronger for the field galaxies than the LSC galaxies.

Similar to the subsamples RV4, RV5 and RV6, the subsamples RV8 and RV9 compensate each other to give $\Delta<5 \%$. A significant dominance of trailing structural modes is noticed for the galaxies that have RVs 4000 to $4200 \mathrm{~km} \mathrm{~s}^{-1}$. The galaxies having $15 \leq m \leq 16$ show an excess number of leading arm galaxies (Table 2).

No preferred structural modes are noticed for 20 subsamples, suggesting the existence of chiral symmetry in the field galaxies. Thus, it seems that the chiral symmetry of field galaxies is independent of the inclination angle, RVs, magnitude and the size of the galaxies.

\section{3. $L S C+$ nearby $L S C$ galaxies $\left(R V \leq 5000 \mathrm{~km} \mathrm{~s}^{-1}\right)$}

The leading and trailing arm galaxies that have $\mathrm{RV} \leq$ $5000 \mathrm{~km} \mathrm{~s}^{-1}$ show the $\Delta$ value merely $1 \%$ (Table 1 ). This result strongly advocates the existence of chiral symmetry in the large scale structure. For the spirals, the difference between the number of leading and trailing arm galaxies is insignificant $(<0.5 \%)$. Thus, the chiral symmetry is found to be strong for the spirals.

A significant dominance $(\Delta>10 \%)$ of trailing structural modes is noticed in the barred spirals. Thus, the violation of chirality in the SB galaxies can be suspected.

No significant dominance $(\Delta> \pm 10 \%)$ of either face-on or edge-on leading or trailing arm galaxies is noticed when the
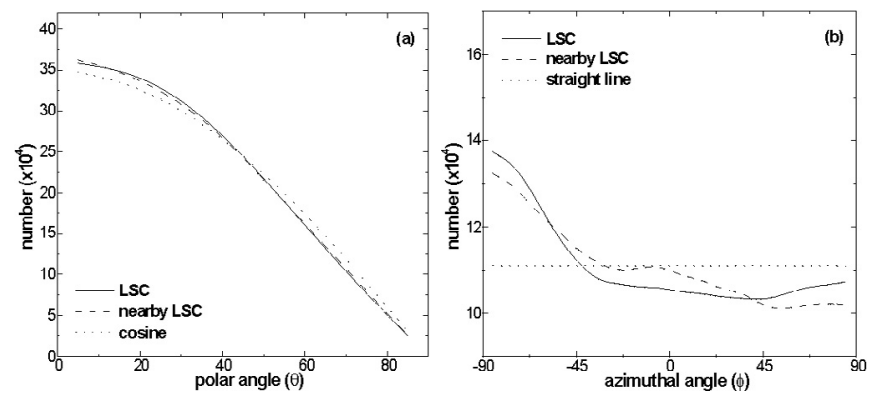

Fig. 5. The expected polar $(\theta)$ a) and azimuthal angle $(\phi)$ distribution b). The solid and the dashed line represent the expected distribution curves for LSC $\left(\mathrm{RV} \leq 3000 \mathrm{~km} \mathrm{~s}^{-1}\right)$ and nearby LSC $\left(3000<\mathrm{RV}\left(\mathrm{km} \mathrm{s}^{-1}\right)\right.$ $\leq 5000$ ) galaxies. The dotted line represents expected distribution when there are no selections on inclination angle and positions.

database of LSC (Aryal et al. 2007a) and nearby LSC galaxies was added (Table 2). A dominance of trailing and leading modes are noticed for the low $(m<13)$ and high magnitude $(m<15)$ galaxies, respectively. Similarly, a dominance $(\Delta>-5 \%)$ of trailing modes is noticed for the galaxies that have $a>3 \mathrm{arcmin}$.

In Fig. 4, we can see the convergence of the scatter plots when moving from LSC subsamples (solid circle) to the LSC + nearby LSC subsamples (solid triangle). This suggests the importance of good statistics in the study of chirality of galaxies. In addition, violation of chirality can be suspected more in bounded system (clusters, e.g. LSC) than in the open system (field, e.g. nearby LSC).

We checked the effect of random distribution by repeating it five more times. Interestingly, the results for all subsamples agree within $\pm 5 \%$ error limit. This consistency advocates our assumption regarding the direction of rotation of the galaxy for an observer.

\section{Preferred alignment}

We assumed spatial isotropic distribution and supergalactic system as a theoretical reference for LSC, nearby LSC and total $(\mathrm{LSC}+$ nearby LSC) galaxies. This spatial reference distribution gives further reference distributions for the polar $(\theta)$ and azimuthal angles $(\phi)$ with respect to the LSC plane.

Aryal \& Saurer (2000) concluded that the expected isotropic $\theta$ and $\phi$-distribution curves change with positions (longitude, latitude) when making selections on the inclination angle. A number of edge-on and face-on galaxies are missing in our database (Figs. 3c, d). We have taken this fact in the simulation and found different expected $\theta$ and $\phi$-distribution curves for LSC and nearby LSC galaxies (Fig. 5). The isotropic distribution curves are based on calculations including $10^{6}$ virtual galaxies.

As a next step, our observations was compared with these isotropic distributions in both the polar $(\theta)$ and azimuthal angle $(\phi)$. For this comparison we used three different statistical tests: chi-square, auto-correlation and the Fourier.

The bin size was chosen to be $10^{\circ}$ for $\theta$ and $20^{\circ}$ for $\phi$ distributions in the statistics. The ranges for the angles $\theta$ and $\phi$ are $0^{\circ}$ to $90^{\circ}$ and $-90^{\circ}$ to $+90^{\circ}$, respectively. The statistically poor bins (number of solution $<5$ ) are omitted in the analysis. The conditions for anisotropy are the following: the chi-square probability $P\left(>\chi^{2}\right)<0.050$, correlation coefficient $C / \sigma(C)> \pm 1$, first order Fourier coefficient $\Delta_{11} / \sigma\left(\Delta_{11}\right)> \pm 1$, and the first order Fourier probability $P\left(>\Delta_{1}\right)<0.150$, as used by Aryal \& Saurer (2004).

Table 3 lists the statistical parameters for the leading and trailing subsamples of LSC, nearby LSC and total galaxies. 
Table 3. Statistics of the polar $(\theta)$ and azimuthal angle $(\phi)$ distribution of leading and trailing patterns in the LSC, nearby LSC and total galaxies (first column). The second and third columns give the chi-square probability $\left(P\left(>\chi^{2}\right)\right)$ and correlation coefficient $(C / C(\sigma))$ for leading arm galaxies. The next two columns list the values of first order Fourier coefficient $\left(\Delta_{11} / \sigma\left(\Delta_{11}\right)\right)$ and first order Fourier probability $P\left(>\Delta_{1}\right)$ for leading arm galaxies. The last four columns repeat the previous columns for the trailing arm galaxies.

\begin{tabular}{|c|c|c|c|c|c|c|c|c|}
\hline \multicolumn{9}{|l|}{ Polar angle $(\theta)$} \\
\hline \multirow[t]{2}{*}{ sample } & \multicolumn{3}{|c|}{ (Leading) } & \multicolumn{5}{|c|}{ (Trailing) } \\
\hline & $P\left(>\chi^{2}\right)$ & $C / C(\sigma)$ & $\Delta_{11} / \sigma\left(\Delta_{11}\right)$ & $P\left(>\Delta_{1}\right)$ & $P\left(>\chi^{2}\right)$ & $C / C(\sigma)$ & $\Delta_{11} / \sigma\left(\Delta_{11}\right)$ & $P\left(>\Delta_{1}\right)$ \\
\hline \multicolumn{9}{|l|}{ LSC galaxies } \\
\hline total (T1) & 0.919 & -0.2 & -0.2 & 0.981 & 0.964 & -0.4 & +0.0 & 0.993 \\
\hline spiral (S1) & 0.650 & +1.2 & -1.6 & 0.235 & 0.960 & -0.1 & -0.6 & 0.799 \\
\hline barred spiral (SB1) & 0.672 & +0.7 & -0.6 & 0.649 & 0.993 & -0.1 & +0.5 & 0.834 \\
\hline \multicolumn{9}{|c|}{ nearby LSC galaxies } \\
\hline spiral (S2) & 0.989 & +0.1 & +0.1 & 0.995 & 0.997 & $\begin{array}{l}-0.2 \\
+0.1\end{array}$ & $\begin{array}{l}+0.2 \\
-0.2\end{array}$ & 0.967 \\
\hline barred spiral (SB2) & 0.043 & +3.3 & -3.1 & 0.003 & 0.003 & +5.2 & -3.7 & 0.001 \\
\hline \multicolumn{9}{|c|}{ LSC + nearby LSC } \\
\hline total (T3) & 0.552 & -0.3 & +0.5 & 0.894 & 0.948 & -0.4 & +0.2 & 0.969 \\
\hline spiral (S3) & 0.971 & +0.2 & -0.8 & 0.716 & 0.992 & -0.1 & -0.4 & 0.902 \\
\hline barred spiral (SB3) & 0.046 & +2.8 & -2.7 & 0.009 & 0.127 & +2.6 & -2.6 & 0.026 \\
\hline others $(\mathrm{O} 3)$ & 0.547 & +0.9 & +0.3 & 0.691 & 0.883 & +0.3 & -0.8 & 0.735 \\
\hline \multicolumn{9}{|l|}{ azimuthal angle $(\phi)$} \\
\hline \multicolumn{9}{|l|}{ LSC galaxies } \\
\hline total (T1) & 0.492 & +1.1 & -1.7 & 0.055 & 0.850 & +0.4 & -1.1 & 0.379 \\
\hline spiral (S1) & 0.814 & +0.5 & -1.5 & 0.229 & 0.953 & +0.2 & -0.9 & 0.635 \\
\hline barred spiral (SB1) & 0.947 & +0.2 & +0.7 & 0.664 & 0.893 & -0.1 & +0.7 & 0.664 \\
\hline \multicolumn{9}{|l|}{ nearby LSC galaxies } \\
\hline total (T2) & 0.839 & +0.5 & +0.7 & 0.664 & 0.993 & +0.2 & +0.7 & 0.737 \\
\hline spiral (S2) & 0.980 & +0.3 & +1.1 & 0.495 & 0.985 & +0.2 & +1.1 & 0.562 \\
\hline barred spiral (SB2) & 0.048 & +2.6 & +3.6 & 0.002 & 0.040 & +1.1 & +2.2 & 0.080 \\
\hline \multicolumn{9}{|c|}{ LSC + nearby LSC } \\
\hline total (T3) & 0.972 & +0.2 & -1.3 & 0.457 & 0.994 & +0.0 & +0.0 & 0.728 \\
\hline spiral (S3) & 0.985 & +0.0 & +0.3 & 0.946 & 0.996 & +0.0 & +0.5 & 0.869 \\
\hline barred spiral (SB3) & 0.095 & +2.5 & +3.2 & 0.005 & 0.401 & +0.8 & +1.7 & 0.194 \\
\hline others $(\mathrm{O} 3)$ & 0.598 & -0.9 & +0.8 & 0.698 & 0.842 & -0.3 & +0.3 & 0.869 \\
\hline
\end{tabular}

\subsection{LSC galaxies $\left(R V \leq 3000 \mathrm{~km} \mathrm{~s}^{-1}\right)$}

In the polar angle $(\theta)$ distribution, all three statistical tests show isotropy for both the leading and trailing pattern LSC galaxies (Table 3). A good agreement between the expected and observed distribution can be seen in Fig. 6a. Thus, no preferred alignment is found in the SV orientations of leading and trailing galaxies that have $\mathrm{RV}<3000 \mathrm{~km} \mathrm{~s}^{-1}$. In the azimuthal angle distribution, a hump at $-80^{\circ}(>1.5 \sigma)$ can be seen for the trailing mode (Fig. 6b). This hump causes the Fourier parameters $\Delta_{11} / \sigma\left(\Delta_{11}\right)$ and $P\left(>\Delta_{1}\right)$ to be -1.7 and $5.5 \%$, respectively. These values suggest a preferred alignment: the SV projections of galaxies tend to orient tangentially with respect to the Virgo cluster center. In the leading mode, a hump at $60^{\circ}(<1.5 \sigma)$ is not sufficient to make the statistics anisotropy (Fig. 6b). Thus, we conclude a random alignment for leading patterns of LSC galaxies in the $\phi$-distribution.

The trailing arm spirals show isotropy in both the $\theta$ and $\phi$-distributions. No humps and dips can be seen (Table 3, Figs. $6 \mathrm{c}, \mathrm{d}$ ). The $\Delta_{11}$ is found $\geq 1.5 \sigma$ for leading arm spirals in both the $\theta$ and $\phi$-distribution. However, the $P\left(>\Delta_{1}\right), P\left(>\chi^{2}\right)$ and $C / C(\sigma)$ values are found well within the error limit (Table 3 ). Hence, weak anisotropy can be suspected in the leading pattern spirals whereas no preferred alignment is noticed in the trailing patterns.

The leading and trailing spiral barred galaxies in the LSC show isotropy in both the $\theta$ and $\phi$-distributions. A very good agreement between the observed and expected distribution can be seen in Figs. 6e, f. Thus, the trailing pattern spirals and barred spirals that have $\mathrm{RV}<3000 \mathrm{~km} \mathrm{~s}^{-1}$ show a random orientation in both the $\theta$ and $\phi$-distributions.

\subsection{Nearby LSC galaxies $\left(3000<R V\left(\mathrm{~km} \mathrm{~s}^{-1}\right) \leq 5000\right)$}

All three statistical tests suggest isotropy for both the leading and trailing arm galaxies that have RVs in the range $3000 \mathrm{~km} \mathrm{~s}^{-1}$ to $5000 \mathrm{~km} \mathrm{~s}^{-1}$ in both the $\theta$ and $\phi$-distributions (Table 3 ). No deviation from the expected distribution can be seen (Figs. 7a, b). Spirals show a similar result. Thus, a random spatial orientation is noticed for the total and spiral galaxies.

The spiral barred galaxies show a preferred alignment in both the leading and trailing structural modes. In the leading SB galaxies, dips at $5^{\circ}(>1.5 \sigma)$ and $15^{\circ}(>1.5 \sigma)$ and humps at $55^{\circ}(\sim 1.5 \sigma)$ and $65^{\circ}(>1.5 \sigma)$ can be seen (Fig. 7e). Similarly, dips at $5^{\circ}(>1.5 \sigma)$ and $15^{\circ}(>1.5 \sigma)$ and humps at $65^{\circ}(\sim 2 \sigma)$ and $75^{\circ}(>2 \sigma)$ can be seen in the $\theta$-distribution of trailing arm SB galaxies (Fig. 7e). These humps and dips cause the SB subsamples to give the $\Delta_{11}$ value $\geq 3 \sigma$. Thus, the SVs of leading and trailing arm SB galaxies tend to be oriented perpendicular the LSC plane. In the $\phi$-distribution, all three statistical tests show anisotropy in both the leading and trailing arm SB galaxies. A similar preferred alignment is noticed in these two structural modes: SV projections tend to point towards the LSC center (or Virgo cluster center). 

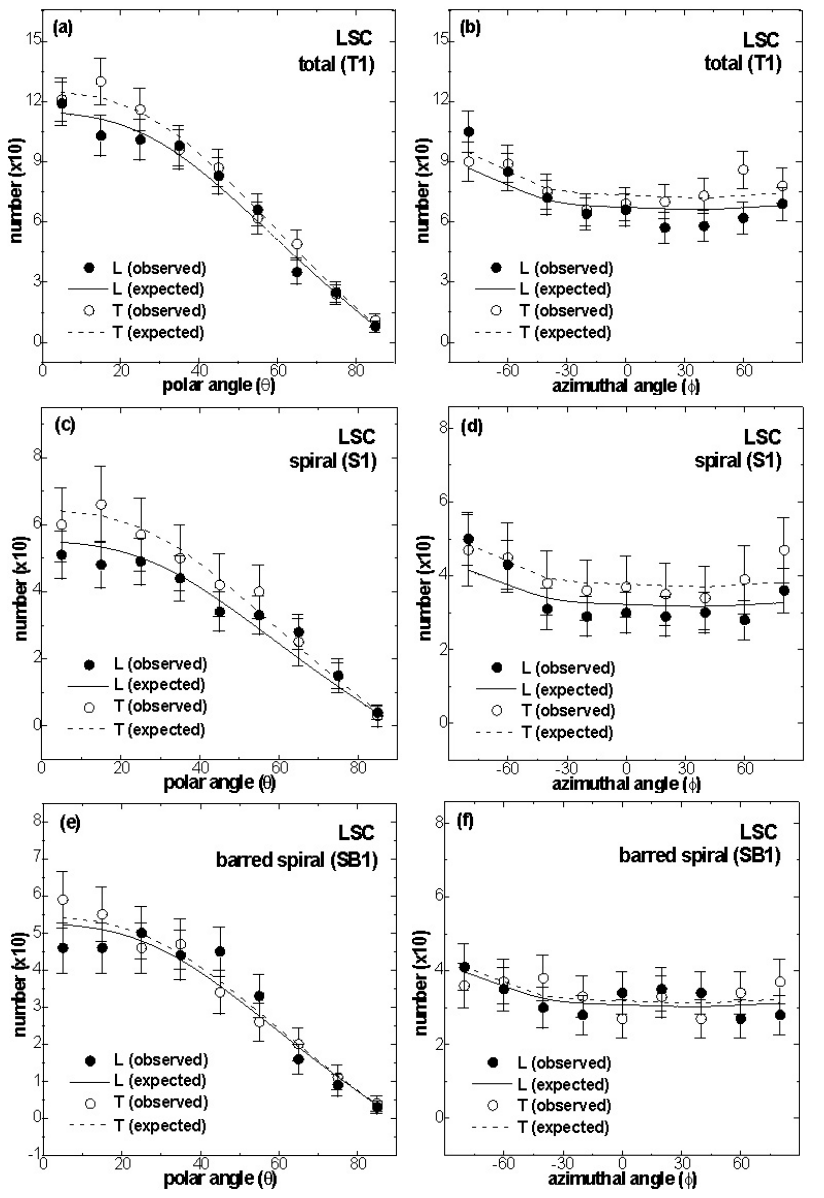

Fig. 6. The polar $(\theta)$ and azimuthal angle $(\phi)$ distribution of leading and trailing patterns in the LSC $\left(\mathrm{RV} \leq 3000 \mathrm{~km} \mathrm{~s}^{-1}\right)$ galaxies. The symbols "•" (solid circle) and "o" (hollow circle) represent the observed distribution of the leading and trailing patterns, respectively. The statistical $\pm 1 \sigma$ error bars are shown. The solid and dashed line represent the expected distribution for leading and trailing type galaxies, respectively. In the histogram, $\theta=0^{\circ}\left(90^{\circ}\right)$ means the $\mathrm{SV}$ of galaxies tend to be oriented parallel (perpendicular) with respect to the LSC. For azimuthal angle, $\phi=0^{\circ}$ means the SV projections tend to direct towards the LSC center (or Virgo cluster center).

\section{3. $L S C+$ nearby $L S C$ galaxies $\left(R V \leq 5000 \mathrm{~km} \mathrm{~s}^{-1}\right)$}

All three statistical tests show isotropy in both the $\theta$ and $\phi$-distributions of leading and trailing arm total galaxies. No significant dips and humps are seen in Figs. 8a, b. Thus, a random orientation of SVs of leading and trailing arm galaxies that have $\mathrm{RV} \leq 5000 \mathrm{~km} \mathrm{~s}^{-1}$ is noticed.

The leading and trailing arm spirals show no preferred alignment in both the $\theta$ and $\phi$-distributions. The observed distribution is found to be consistent with the expected distribution (Figs. 8c, d). All statistical parameters advocate isotropy. Thus, we conclude no preferred alignment in the leading and trailing arm spirals.

In the polar angle distribution, a similar deviation can be seen in the leading and trailing arm spiral barred galaxies (Fig. 8e). All three statistical tests suggest anisotropy (Table 3). The $\Delta_{11} / \sigma\left(\Delta_{11}\right)$ is negative $(>2 \sigma)$ for both structural modes, suggesting a similar preferred alignment: the $\mathrm{SV}$ s of galaxies tend to be oriented perpendicular to the LSC plane. In the azimuthal angle distribution, the chi-square probability $\left(P\left(>\chi^{2}\right)\right)$ is found to be greater than $5 \%$ significance level in both structural modes. The auto-correlation coefficient suggests anisotropy $(>2 \sigma)$ for
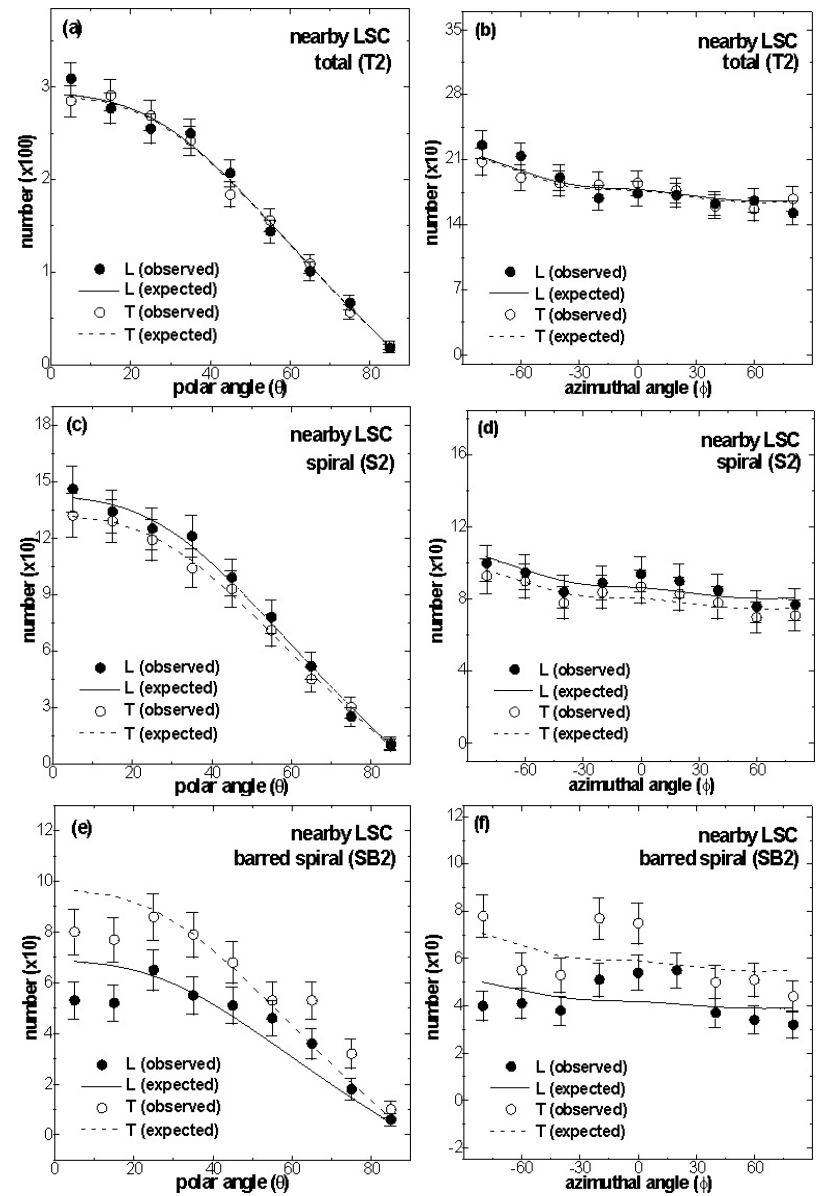

Fig. 7. The polar $(\theta)$ and azimuthal angle $(\phi)$ distributions of leading and trailing pattern in the nearby LSC $\left(3000<\mathrm{RV}\left(\mathrm{km} \mathrm{s}^{-1}\right) \leq 5000\right)$ galaxies. Symbols as in Fig. 6.

the leading mode and isotropy for the trailing mode. The Fourier probability $\left(P\left(>\Delta_{1}\right)\right)$ is $<5 \%$ for the leading mode. In this mode, the $\Delta_{11}$ is found to be positive at $>3 \sigma$ level, indicating a preferred alignment. Thus, the SV projections of leading arm SB galaxies tend to direct towards the LSC center. A significant hump at $0^{\circ}$ supports this result (Fig. 8f).

No preferred alignment is noticed for morphologically unidentified galaxies (subsample O3). All statistical parameters indicate isotropy. However, the statistics is not rich $(<100)$ in this subsample.

\section{Discussion}

Figure 9a shows a comparison between the $\Delta \%$ and the $\Delta_{11} / \sigma\left(\Delta_{11}\right)$ value of leading and trailing arm galaxies in the total sample and subsamples. This plot reveals the correlation between the chirality (non-chirality) and the random (preferred) alignment of galaxies in the total sample and subsamples. The grey-shaded region represents the coexistence between the chirality and the random orientation of galaxies.

In the $\theta$-distribution, $\Delta_{11} / \sigma\left(\Delta_{11}\right)$ values of the total samples (T1, T2 and T3) lie in the grey-shaded region, suggesting no preferred alignment. In addition, the $\Delta \%$ values for the total sample (T1, T2 and T3) are well within the 5\% error limit (grey-shaded region). Thus, a good correlation between the random alignment and the chiral property is found in the total leading and trailing arm galaxies. 

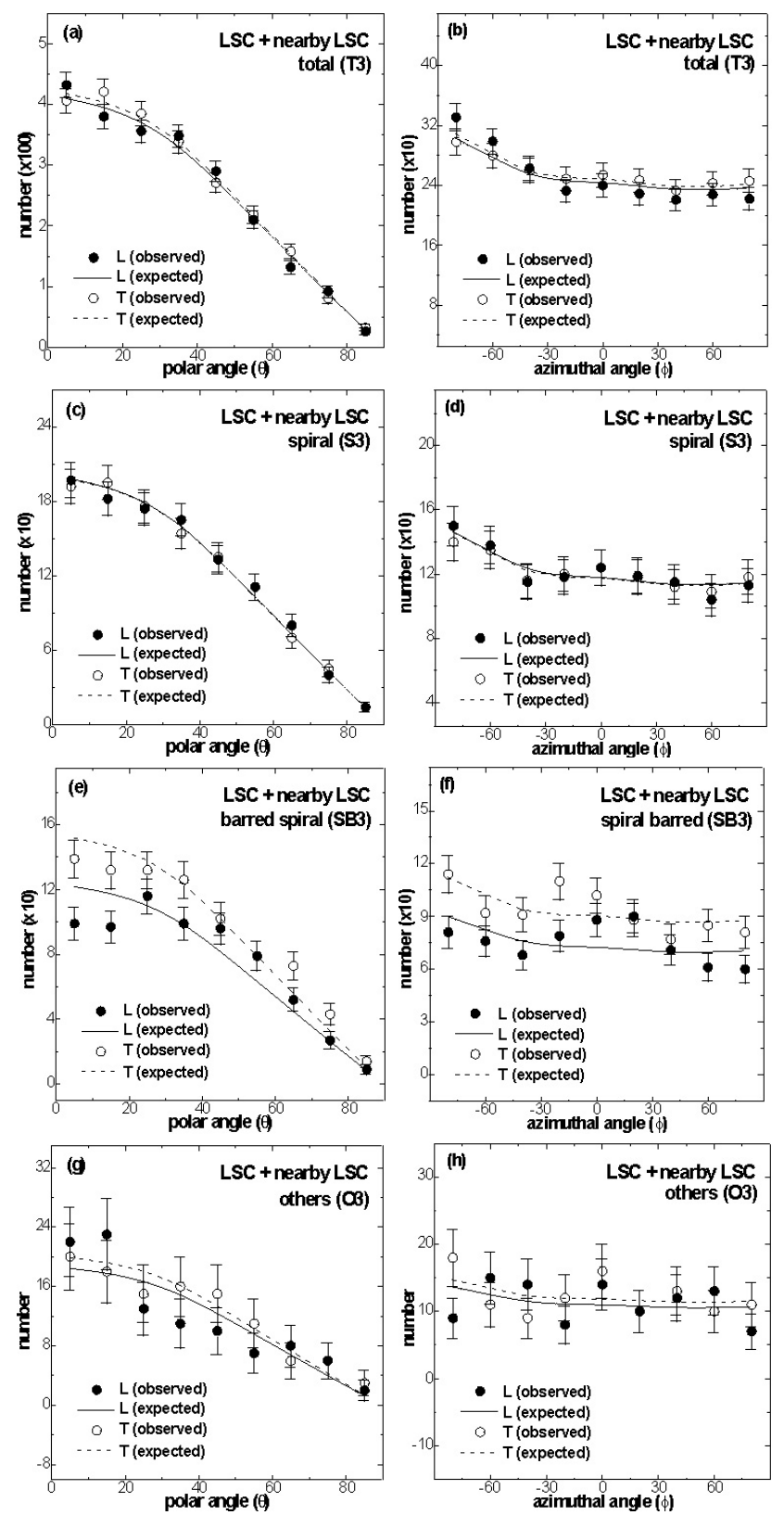

Fig. 8. The polar $(\theta)$ and azimuthal angle $(\phi)$ distributions of leading and trailing pattern in the total (LSC + nearby LSC) galaxies. Symbols as in Fig. 6.

Two spiral subsamples, S2 and S3, show a similar trend to the total sample. Interestingly, two SB subsamples, SB2 and SB3, show an opposite trend to the total sample. The $\Delta_{11} / \sigma\left(\Delta_{11}\right)$ and $\Delta \%$ values of these subsamples (SB2, SB3) cannot be seen in the grey-shaded region, suggesting a preferred alignment (Fig. 9a). Thus, a good correlation between the preferred alignment and the achiral (non-chiral) property is found in the SB subsamples. The subsamples S1 and SB1 show different properties because of the presence of Virgo cluster galaxies in the database (Aryal et al. 2007a).

In the $\phi$-distribution, a good correlation between the $\Delta_{11} / \sigma\left(\Delta_{11}\right)$ and $\Delta \%$ value is found for SB2 and SB3 subsamples. The leading and trailing arm galaxies of these subsamples show a preferred alignment in the spatial orientation and the nonchiral property in the number statistics. Thus, the coexistence of achirality and the preferred alignment of SB galaxies cannot be
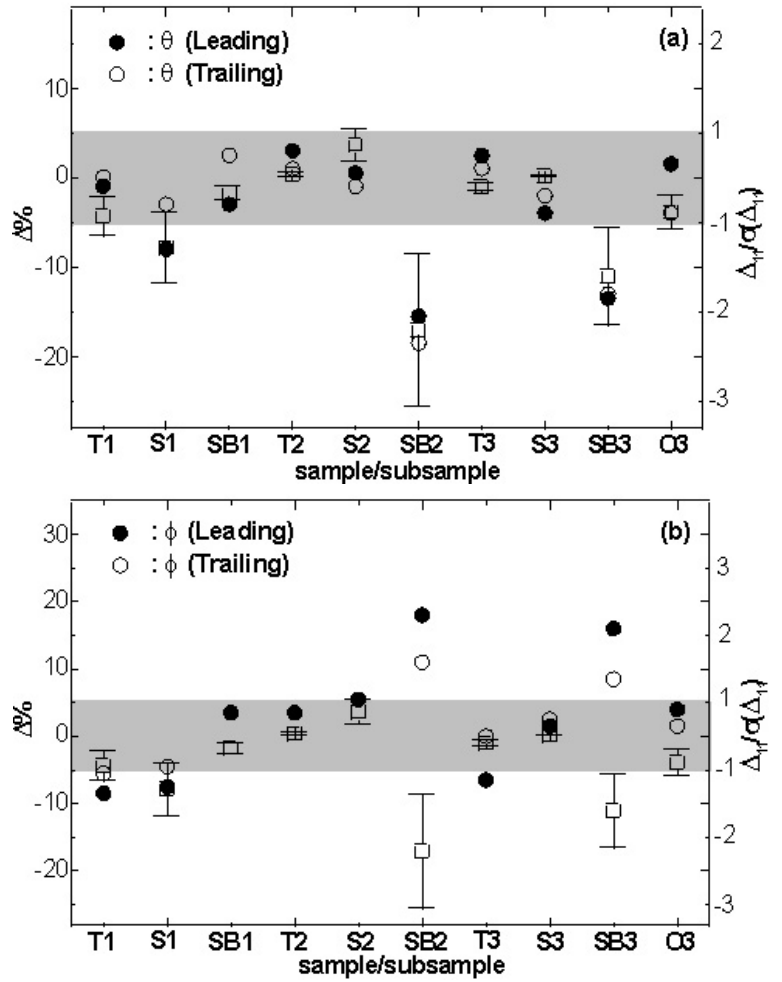

Fig. 9. A comparison between the chiral symmetry $(\Delta \%)$ and the preferred alignment $\left(\Delta_{11} / \sigma\left(\Delta_{11}\right)\right)$ in the polar $(\theta)$ and azimuthal $(\phi)$ angle distribution of leading and trailing samples and subsamples. Error bars as in Fig. 4.

denied. In the leading total subsamples (T1 and T3), a weak preference can be seen in the $\phi$-distribution (Fig. 9b). However, the trailing total subsamples (T1, T2 and T3) show a random orientation of SV projections of galaxies. The subsamples S2, S3 and $\mathrm{O} 3$ show a good correlation between the random alignment and the chiral property (Fig. 9b).

Thus, our results in the $\theta$ and $\phi$-distributions lead us to predict the coexistence of chiral property and the random alignment of galaxies. This coexistence is found to be significant in the spiral galaxies. This result indicates the fact that the chiral property is a global phenomenon rather than a local phenomenon. Interestingly, we noticed coexistence between the preferred alignment and the achiral property in the spiral barred galaxies. It seems that the chirality loss sequence (spiral $\longrightarrow$ barred spiral), as predicted by Capozziello \& Lattanzi (2006), might be true.

Aryal et al. (2007a) found a random alignment of leading and trailing arm LSC galaxies in the two dimensional analysis. We found a similar result in the three dimensional analysis. Aryal et al. (2007a) concluded that the Hubble morphological sequence is related to the degree of chirality of galaxies. Their result hints that the late-type galaxies are chiral objects, while early-type galaxies might be achiral. In this perspective, the Hubble diagram probably represents a chirality-loss sequence.

Aryal \& Saurer $(2004,2005$ b, 2006) and Aryal et al. (2007b) studied the spatial orientation of galaxies in 32 Abell clusters of BM type I (2004), BM type III (2005b), BM type II-III (2006) and BM type II (2007) finding a significant preferred alignment of angular momentum of the galaxies in the late-type cluster (BM type II-III, BM type III). In addition, they noticed that the randomness decreases systematically in galaxy alignments from 
early-type (BM type I, II) to late-type (BM type II-III, III) clusters. Thus, the existence of chirality in BM type I cluster, as predicted by Capozziello \& Lattanzi (2006) might be true. We noticed a very good correlation between the randomness and the chiral symmetry of galaxies. This result indicates that the progressive loss of chirality might have some connection with the rotationally-supported (spirals, barred spirals) and randomized (lenticulars, ellipticals) system. Thus, we suspect that the dynamical processes in the cluster evolution (early-type $\longrightarrow$ latetype) give rise to a dynamical loss of chirality. In other words, existence of non-chirality and non-randomness can be suspected for the late-type cluster galaxies. It would be interesting to test this prediction by analyzing the chiral property of spirals in the late-type clusters in the future.

The "Li model" (Li 1998) predicts that the celestial bodies acquire angular momentum during formation from the global rotation of the Universe. Godlowski et al. (2003) derived a relation between angular momentum and the mass of a galaxy using the "Li model" (Li 1998) and tested this relation for Tully's galaxy group (Godlowski et al. 2005). For that group, no preferred alignment of SVs of galaxies is noticed. They conclude that the minima of the relation between angular momenta and masses of galaxy structures can be verified observationally. In this study, we notice a vanishing angular momentum for the leading and trailing arm galaxies in the LSC. In addition, our result for the leading and trailing arm nearby LSC and total galaxies supports this. This result is interesting in the sense that the chiral property might coexist with the system (huge structures) of vanishing angular momentum.

\section{Conclusion}

We studied the chiral symmetry and the spatial orientation of 2288 galaxies that have RV less than $5000 \mathrm{~km} \mathrm{~s}^{-1}$. These galaxies include LSC $\left(\mathrm{RV}<3000 \mathrm{~km} \mathrm{~s}^{-1}\right)$ and nearby LSC $\left(3000<\mathrm{RV}\left(\mathrm{km} \mathrm{s}^{-1}\right) \leq 5000\right)$ galaxies. We assumed random direction for the rotation of galaxies and classified leading and trailing modes by observing the patterns ( $\mathrm{S}$ - and $\mathrm{Z}$-shaped) of the arms in the spiral and barred spiral galaxies. The distributions of structural modes are studied in the total sample and 69 subsamples. To examine non-random effects, the distribution of SV and SV projections of galaxies in the total sample and 9 subsamples are studied. We compared our results concerning chiral symmetry and the preferred spatial alignments of the leading and trailing modes, and discussed them in the context of Capozziello \& Lattanzi's (2006) prediction. We summarize our result as follows:

1. A good correlation between the random alignment and the chiral symmetry of galaxies is found in the total leading and trailing arm LSC $\left(\mathrm{RV}<3000 \mathrm{~km} \mathrm{~s}^{-1}\right)$, nearby LSC $\left(3000<\mathrm{RV}\left(\mathrm{km} \mathrm{s}^{-1}\right) \leq 5000\right)$ and total (LSC + nearby LSC) galaxies. Spiral galaxies show a similar results as shown by the total sample: chiral symmetry and the random alignment.

2. A preferred alignment of angular momentum vectors of leading and trailing arm SB galaxies is noticed. In addition, chiral symmetry is found to be weak for barred spirals. Thus, we noticed a good correlation between the preferred alignment and non-chiral property in the leading and trailing arm SB galaxies. Our results verify the prediction made by Capozziello \& Lattanzi (2006) concerning chirality loss sequence (spiral $\longrightarrow$ barred spiral).
3. The random alignments of galaxies in BM type I cluster (Aryal \& Saurer 2004) lead us to suspect the existence of chiral symmetry. This was predicted by Capozziello \& Lattanzi (2006). This result reveals that the progressive loss of chirality might have some connection with the rotationally-supported (spirals, barred spirals) and randomized (lenticulars, ellipticals) systems. Thus, we suspect that the dynamical processes in the cluster evolution give rise to a dynamical loss of chirality. In other words, existence of non-chirality and non-randomness can be suspected for the late-type cluster galaxies.

4. Chiral symmetry is found to be strong in 52 subsamples (75\% subsamples). This result leads us to conclude the existence of chiral symmetry between the leading and trailing modes in the LSC, nearby LSC and the total (LSC + nearby LSC).

5. We found a vanishing angular momentum for the leading and trailing arm galaxies in the LSC. In addition, our result for the leading and trailing arm nearby LSC and total (LSC + nearby LSC) galaxies support this. Thus, the chiral symmetry coexists with the system of vanishing angular momentum. Thus, the coexistence between the chiral symmetry and the random orientation of galaxies might have originated from the fact that the celestial bodies acquire angular momentum from the global rotation of the Universe (Li 1998).

A wide discussion is needed regarding the relation between the chirality-loss sequence and the preferred orientation of galaxies. The result of this paper demands a series of work mainly in the clusters. In addition, three dimensional determination of the leading and the trailing arm patterns in the galaxies is a very important problem. The true structural mode of a galaxy must involve a determination of which side of the galaxy is closer to the observer (Binney \& Tremaine 1987). We intend to determine the true structural modes of our galaxies in the future.

Acknowledgements. The authors wish to thank the referee for insightful comments regarding the true structural modes of individual galaxies. This research has made use of the NASA/IPAC Extragalactic Database (NED), which is operated by the Jet Propulsion Laboratory, California Institute of Technology, under contract with the National Aeronautics and Space Administration. We acknowledge Profs. Lok Narayan Jha, Udayraj Khanal and Mukunda Mani Aryal for insightful discussions.

\section{Appendix A}

We adopt the method described by Flin \& Godlowski (1986) to calculate the polar $(\theta)$ and azimuthal $(\phi)$ angle of galaxies. The polar angle $(\theta)$ represents the angle between the galactic SV and a reference plane. The angle between the projection of a galactic $\mathrm{SV}$ on to this reference plane is the azimuthal angle $(\phi)$. The reference plane is the LSC plane. The formulae to obtain $\theta$ and $\phi$, as given in Flin \& Godlowski (1986), are as follows:

$\sin \theta=-\cos i \sin B \pm \sin i \sin P \cos B$

$\sin \phi=(\cos \theta)^{-1}[-\cos i \cos B \sin L+\sin i$

$\mp \sin P \sin B \sin L \mp \cos P \cos L]$

where $L, B$ and $P$ are the supergalactic longitude, latitude and position angle, respectively. The angle $i$ is the inclination angle, estimated with Holmberg's (1946) formula: $\cos ^{2} i=\left[(b / a)^{2}-\right.$ $\left.q^{2}\right] /\left(1-q^{2}\right)$ where $b / a$ is the measured axial ratio and $q$ is the intrinsic flatness of disk galaxies. We adopt the supergalactic coordinate system as defined by Tammann \& Sandage (1976). 


\section{Appendix B}

Aryal \& Saurer $(2000,2001)$ studied the results of random simulations for the expected isotropic distributions of the polar and azimuthal angles of galaxy rotation axes. They found that any selection criteria imposed on the data may cause severe changes in the shapes of the expected isotropic distribution curves. In order to remove the selection effects concerning positions and inclination angles we use the method described by Aryal \& Saurer (2000, 2001). In their method, the spatial distribution of galaxy rotation axes is assumed to be isotropic. Then, due to projection effects, $i$ is distributed $\propto \sin i, B$ can be distributed $\propto \cos B$, the variables $L$ and $P$ can be distributed randomly, and Eqs. (1) and (2) are used to calculate the corresponding values of $\theta$ and $\phi$.

To remove the selection effect concerning nearly face-on galaxies, the inclination angle was randomly distributed $\propto$ sine in the range $25^{\circ}$ to $85^{\circ}$ in the numerical simulation. The isotropic distribution curves are based on calculations including $10^{6}$ virtual galaxies.

The expected isotropic distribution curves of $\theta$ and $\phi$ of our galaxies are shown in Fig. 5. In this figure, the dotted lines are the expected isotropic distribution curves when there is no selection on positions and inclination angles in the database. The expected $\theta$-distribution curve is found to hardly deviate from the cosine curve. The deviation at small angles $\left(<15^{\circ}\right)$ in Fig. 5a is due to the selections on inclination angles. The expected $\phi$-distribution curve remarkably deviates from the random distribution (Fig. 5b). This deviation is mainly due to the inhomogenous distributions of $L$. The azimuthal angle distribution changes with $L$ even when the range of $i$ and $B$ is full (Aryal \& Saurer 2000).

\section{Appendix C}

We set the chi-square probability $P\left(>\chi^{2}\right)=0.050$ as the critical value to discriminate isotropy from anisotropy (Godlowski 1993). We expect an auto correlation coefficient $C \rightarrow 0$ for an isotropic distribution.

The Fourier test is useful when the deviation from isotropy is slowly varying with the angles (in our case, polar and azimuthal angles).

A method of expanding a function by expressing it as an infinite series of periodic functions (sine and cosine) is called a Fourier series. Let $N$ denote the total number of solutions for galaxies in the sample, $N_{k}$ the number of solutions in the $k$ th bin, $N_{0}$ the mean number of solutions per bin, and $N_{0 k}$ the expected number of solutions in the $k$ th bin. Then the Fourier series is given by (taking first order Fourier mode),

$$
N_{k}=N_{0 k}\left(1+\Delta_{11} \cos 2 \beta_{k}+\Delta_{21} \sin 2 \beta_{k}+\ldots\right) \text {. }
$$

Here the angle $\beta_{k}$ represents the polar angle in the $k$ th bin. The Fourier coefficients $\Delta_{11}$ and $\Delta_{21}$ are the parameters of the distributions. We obtain the following expressions for the Fourier coefficients $\Delta_{11}$ and $\Delta_{21}$,

$$
\begin{aligned}
& \Delta_{11}=\sum\left(N_{k}-N_{0 k}\right) \cos 2 \beta_{k} / \sum N_{0 k} \cos ^{2} 2 \beta_{k} \\
& \Delta_{21}=\sum\left(N_{k}-N_{0 k}\right) \sin 2 \beta_{k} / \sum N_{0 k} \sin ^{2} 2 \beta_{k} .
\end{aligned}
$$

The standard deviations $\left(\sigma\left(\Delta_{11}\right)\right)$ and $\left(\sigma\left(\Delta_{21}\right)\right)$ can be estimated using the expressions,

$$
\begin{aligned}
& \sigma\left(\Delta_{11}\right)=\left(\sum N_{0 k} \cos ^{2} 2 \beta_{k}\right)^{-1 / 2} \\
& \sigma\left(\Delta_{21}\right)=\left(\sum N_{0 k} \sin ^{2} 2 \beta_{k}\right)^{-1 / 2} .
\end{aligned}
$$

The probability that the amplitude

$\Delta_{1}=\left(\Delta_{11}^{2}+\Delta_{21}^{2}\right)^{1 / 2}$

is greater than a certain chosen value is given by the formula

$P\left(>\Delta_{1}\right)=\exp \left(-n N_{0} \Delta_{1}^{2} / 4\right)$

with standard deviation

$\sigma\left(\Delta_{1}\right)=\left(2 / n N_{0}\right)^{1 / 2}$.

The Fourier coefficient $\Delta_{11}$ gives the direction of departure from isotropy. The first order Fourier probability function $P\left(>\Delta_{1}\right)$ estimates whether (smaller value of $P\left(>\Delta_{1}\right)$ or not (higher value of $P\left(>\Delta_{1}\right)$ a pronounced preferred orientation occurs in the sample.

\section{References}

Abell, G. O., Corwin, H. G., \& Olowin, R. P. 1989, ApJS, 70, 1 Aryal, B., \& Saurer, W. 2000, A\&A, 364, L97 (AS)

Aryal, B., \& Saurer, W. 2001, in Galaxy Disk and Disk Galaxies, ed. J. S. Funes, \& E. M. Corsini, ASP Conf. Ser., 230, 145

Aryal, B., \& Saurer, W. 2004, A\&A, 425, 871

Aryal, B., \& Saurer, W. 2005a, A\&A, 432, 841

Aryal, B., \& Saurer, W. 2005b, A\&A, 432, 431

Aryal, B., \& Saurer, W. 2006, MNRAS, 366, 438

Aryal, B., Acharya, S., \& Saurer, W. 2007a, Ap\&SS, 307, 4, 369

Aryal, B., Paudel, S., \& Saurer, W. 2007b, MNRAS, 379, 1011

Bagchi, M., Ray, S., Dey, M., \& Dey, J. 2006, A\&A, 450, 431

Binney, J., \& Tremaine, S. 1987, in Galactic Dynamics (Princeton New Jersey: Princeton Univ. Press)

Brunzendorf, J., \& Meusinger, H. 1999, A\&ASS, 139, 141

Capozziello, S., \& Lattanzi, A. 2006, Ap\&SS, 301, 189

Chang, L., et al. 2007, Phy. Rev. C, 75, 015201

Corwin, H. G., de Vaucouleurs, A., \& de Vaucouleurs, G. 1985, in Univ. Texas Monogr. Astron., 4, 1

de Vaucouleurs, G., de Vaucouleurs, A., Corwin, et al. 1991, Third Reference Catalogue of Bright Galaxies (New York: Springer-Verlag)

Fall, S. M. 1992, in Progress in Cosmology, Proc. of the Oxford Intl. Symp. (Oxford, Dordrecht: D. Reidel Publishing Co.), 347

Flin, P., \& Godlowski, W. 1986, MNRAS, 222, 525

Garcia-Garcia, A. M., \& Cuevas, E. 2006, Phy. Rev. B., 74, 113101

Godlowski, W. 1993 MNRAS, 265, 874

Godlowski, W., Szydlowski, M., Flin, P., \& Biernacka, M. 2003, J. General Relativity \& Gravitation, 35, 907

Godlowski, W., Szydlowski, M., \& Flin, P. 2005, J. General Relativity \& Gravitation, 37, 615

Haynes, M., \& Giovanelli, R. 1984, AJ, 89, 6, 758

Holmberg, E. 1946, Medd. Lund. Astron. Obs., Ser VI, No. 117

Kodaira, K., Okamura, S., \& Ichikawa, S. 1990, Photometric Atlas of Northern Bright Galaxies (Tokyo: Univ. of Tokyo Press) (PANBG)

Lauberts, A. 1982, ESO/Uppsala Survey of the ESO B Atlas, ESO (Garching bei Muenchen)

Li-Xin, Li. 1998, J. General Relativity \& Gravitation, 30, 497

Liddle, A. R., \& Lyth, D. H. 2000, Cosmological Inflation and Large-Scale Structure (Cambridge: Cambridge Univ. Press)

Nilson, P. 1973, Uppsala General Catalogue of Galaxies, Nova Acta Uppsala University, Ser. V:A, Vol. 1 (UGC)

Nilson, P. 1974, Upps. Astron. Obs. Rep., 5 (UGCA)

Oort, J. H. 1970a, Science, 170, 1363

Oort, J. H. 1970b, A\&A, 7, 405

Olling, R. P., \& Merrifield, M. R. 2000, MNRAS, 311, 361

Ostriker, J. P., Peebles, P. J. E., \& Yahil, A. 1974, ApJ, 193, L1

Pasha, I. I. 1985, Sov. Astron. Lett., 11, 1

Peacock, J. A. 1999, in Cosmological Physics (Cambridge: Cambridge Univ. Press)

Shectman, S. A., Landy, S. D., Oemler, A., et al. 1996, AJ, 470, 172

Struble, M.F., \& Rodd, H. J. 1999, ApJS, 125, 355

Sugai, H., \& Iye, M. 1995 MNRAS, 276, 327

Tammann, G. A., \& Sandage, A. 1976, ApJ, 207, L1

Thomasson, M., Donner, K. J., Sundelius, B., et al. 1989, A\&A, 211, 25 